\title{
Prevention of uterine perforation during intracavitary brachytherapy of cervical cancer
}

\author{
Mehmet Bayrak, MD', Candan D. Abakay, MD² \\ 'Department of Gynecologic Oncology, Uludag University Hospital, Bursa, Turkey, ${ }^{2}$ Department of Radiation Oncology, Uludag University \\ Hospital, Bursa, Turkey
}

\begin{abstract}
Purpose: Intracavitary brachytherapy (ICBT) is a part of standard treatment for loco-regionally advanced cervical cancers. ICBT requires a tandem applicator insertion through cervical canal into uterine cavity. Accurate placement through cervical canal, which is distorted by cancer, is crucial to successful treatment. The objective of this study was to investigate actual complication rate of a Smit sleeve insertion performed by experienced gynecologists in a tertiary referral center.

Material and methods: Clinical data of 328 patients with cervical cancer treated using ICBT, between January 2013 and August 2019, were retrospectively evaluated. Predisposing factors that could have increased the risk of uterine perforation were recorded. Pre-operative ultrasound was carried out for visualization of uterine curvature and selection of an appropriate Smith sleeve length. All applications were performed by a gynecologic oncology fellow or an expert gynecologist.

Results: 317 patients were suitable for analysis. Only one $(0.3 \%)$ applicator placement resulted in uterine perforation. In two patients, Smit sleeve dislocated after first brachytherapy and reinserted. Adequate applicator placement was achieved, and treatment was completed as planned in 316 cases.

Conclusions: A cervical sleeve technic, which reduced the need for multiple insertions and placement of this instrument by an expert gynecologist minimize the risk of complication relative to historical controls.

J Contemp Brachytherapy 2021; 13, 2: 167-171 DOI: https://doi.org/10.5114/jcb.2021.105284
\end{abstract}

Key words: uterine perforation, cervical cancer, brachytherapy, Smit sleeve.

\section{Purpose}

Intracavitary brachytherapy is a part of standard treatment for loco-regionally advanced stage cervical cancers, typically in combination with external beam radiotherapy and chemotherapy. External beam radiotherapy is administered first to decrease the volume of primary tumor. Then, intracavitary brachytherapy is used to boost the dose delivered to affected area while sparing the surrounding normal tissue. Intracavitary brachytherapy is also administered in an effort to maximize local control [1]. It has been demonstrated that the prognosis is more favorable with concurrent platinum-based chemoradiation and brachytherapy in terms of overall survival, progression-free survival, and local and distant recurrences $[2,3]$.

Intracavitary brachytherapy can be delivered with either low-dose-rate ( 0.4 to 2 Gy per hour), pulsed-doserate, or high-dose-rate (> 12 Gy per hour) methods. Lowdose-rate brachytherapy requires one or two insertions, but high-dose-rate is predominantly administered using three to six insertions, which varies between centers.
Both methods can be initiated near or after the completion of external beam radiotherapy. High- and low-dose rates appear to have similar efficacy and late complications outcomes in the management of cervical carcinoma, as suggested by a Cochrane database meta-analysis [4]. However, they recommended the use of high-dose-rate brachytherapy due to various potential advantages (rigid immobilization, patient convenience, outpatient treatment, and individualized treatment).

On the other hand, brachytherapy is requiring an invasive procedure to be carried out. Regional or general anesthesia is needed for relaxation and dilatation of the endocervical canal to insert tandem applicator into the uterine cavity through the endocervical os. However, inserting a tandem into a cervix with cancer may create complications, such as vaginal laceration and uterine perforation. In the literature, uterine perforation rates due to tandem applicator insertion were reported ranging from 1.4-14.6\% [5-7]. Uterus perforation may cause tumor spreading to abdominal cavity. In addition, in case of undetected perforation and treatment initiation, this may lead to an increased risk of delayed radiation injury 
to adjacent pelvic structures. Therefore, accurate application placement is necessary to avoid worse treatment outcomes [7].

In order to facilitate multiple insertions of fractionated therapy, the use of a cervical sleeve that enables repeated tandem insertions without repeated general anesthetic exposure has been developed. The cervical sleeve technique is an application that is not mandatory for brachytherapy but facilitates the procedure. The Smit sleeve is a plastic tube with drainage holes that are fixed with stitches to the ectocervix or upper vagina at the time of the first applicator insertion. It helps to keep the cervical canal dilated for subsequent applicator placements throughout the course of treatment. Additionally, it helps guiding the insertion of tandem applicator without anesthetic. The stent sleeve remains in place for the entire course of brachytherapy and is removed after the last brachytherapy session. The one-time application reduces risks but does not eliminate them. However, it has been reported that the risk of perforation is within the range of 0.3 to 2.6 per 1,000 intrauterine device insertions [8]. At the end of external radiotherapy, the tumor usually shrinks, the cervix typically transforms into a deformed shape, and cervical obliteration is detected in the examination. Because of anatomical deformation, the complication rate is higher even in experienced hands. In our institution, contrary to the majority, the cervical sleeve has been inserted by a gynecologic oncologist. All patients are visualized on ultrasonography to determine whether the sleeve lies within the uterine cavity and to detect any possible perforation in the uterine wall, after which, the patient is transferred into radiation oncology unit.

In this study, we aimed to present the role and benefits of the Smit cervical sleeve technique applied by a gynecologic oncologist in an attempt to minimize the risk of complications.

\section{Material and methods}

A retrospective study was conducted including 328 cases of cervical cancer, treated with external beam radiotherapy and brachytherapy at the Radiation Oncology Unit, Uludağ University Hospital, between January 2013 and August 2019. The study protocol was approved by the Uludağ University institutional ethics board (2019-14/26).

Patient data were collected from the hospital records and operative notes. Patients in whom cervical

Table 1. Characteristics of patients

\begin{tabular}{lc}
\hline Variables \\
\hline Age (years), median (range) & $55(27-85)$ \\
\hline Post-menopause $(\%, n)$ & $63.7(202)$ \\
\hline Nulliparity $(\%, n)$ & $5.6(18)$ \\
\hline Stage $(\%, n)$ & \\
\hline I $n$ & $10.7(34)$ \\
\hline II & $42.2(134)$ \\
\hline II & $37.2(118)$ \\
\hline
\end{tabular}

cancer was confirmed and were primarily treated with radiotherapy were included in the study. We excluded patients with incomplete medical records. Patients' characteristics and clinical and pathological factors, which could contribute to increased risk of perforation were recorded (Table 1).

Radiotherapy was administrated with volumetric modulated arc therapy (VMAT), followed by a brachytherapy boost. Before the treatment, each patient underwent computed tomography (CT) simulation in a supine position. Gross tumor volume consisted of all areas of gross disease, including the primary tumor and lymph nodes. Clinical target volume included uterus, part of vagina, parametrium, paravaginal soft tissue, and pelvic lymph node regions (i.e., common iliac, internal and external iliac, obturator, and presacral). Planning target volume was generated by a $0.7 \mathrm{~cm}$ expansion of the clinical target volume in all directions. Organs at risk (OARs) involved small intestine, rectum, bladder, and bilateral femoral heads. An external beam radiotherapy consisting of total dose of 50-60 Gy with sequential or simultaneously boost technique to the involved nodes and parametrial space was delivered. The treatment was administered once daily, five times per week using linear accelerators (Elekta). Concurrent weekly cisplatin-based chemotherapy was administered with radiotherapy.

At the end of external beam radiotherapy, high-doserate intracavitary brachytherapy was administered using tandem cylinder applicators after the Smit sleeve was inserted by a gynecologic oncologist. At our center, due to the lack of anesthesia equipment and room in the radiation oncology department, the cervical Smit sleeve is inserted by gynecologic oncologists in the operation room rather than radiation oncologists. Three fractions of $7 \mathrm{~Gy}$ were delivered to point A using a ${ }^{192}$ Ir brachytherapy source. Brachytherapy plan was optimized manually by modifying dwell time and ${ }^{192}$ Ir source positions.

Following the last session of external beam radiotherapy, patients selected for brachytherapy were evaluated in an outpatient gynecology department for clinical examination, and transabdominal and transvaginal ultrasonography was routinely performed to identify any uterine anomalies and to measure cervico-fundal length.

In accordance with our institutional protocols, a cervical sleeve was used to facilitate insertion of the tandem, additionally acting as a stopper for the ring in the cervix during brachytherapy (Figure 1). The Smit sleeve is a plastic tube available in varying lengths from 2 to $8 \mathrm{~cm}$. A flange at the proximal side contains four holes, one with a radiopaque marker and three other holes enabling the sleeve to be sutured in the cervix or, in case of extensive necrotic tumor, to the upper vaginal wall in the lateral fornix. The Smit sleeve is placed in cervico-uterine canal at the time of the first applicator insertion. It maintains the cervix dilated for subsequent applicator insertions throughout brachytherapy. Different types of cervical sleeve modifications have been used in prior studies $[9,10]$. Moreover, there is a dead end in the distal part of cervical sleeve, thus preventing the tandem applicator from passing through the uterine fundus. 


\section{Smit sleeve application technique}

The Smit sleeve insertion was undertaken in the operating room with general anesthesia. In case of any significant medical contraindication, a spinal anesthetic was used. The patient was arranged in a lithotomy position, and after placing of speculum, the cervix was identified and a tenaculum was applied on the anterior cervical lip to straighten out the uterine canal. A hysterometry was used to measure the depth of uterine cavity and to determine its position. The decision to use transabdominal ultrasonography was individualized and depended on the hysterometry resistance. The cervical canal was dilated using Hegar dilators up to gauge 7 , and an appropriate length of the sleeve was chosen from the available range $(2,4$, or $6 \mathrm{~cm})$. This was then inserted in the cervical canal and stitched to the 3,9,12 o' clock sites of the cervix with non-absorbable silk sutures. After recovery, the patients were observed for four hours and discharged. All the Smit sleeve insertions were performed by a gynecologic oncologist or an expert gynecologist.

The adequacy of sleeve placement was assessed post-operatively with CT imaging in the radiation oncology unit. After three consecutive sessions of high-doserate brachytherapy, the sleeve was removed in the outpatient clinic without anesthetic.

\section{Results}

From January 2013 to August 2019, cervical sleeve insertions were performed in 328 patients. 11 patients were excluded because of missing data. After exclusion, 317 patients were left for analysis.

The median patient age was 55 years (range, 27-85 years), of which, 114 (36\%) were older than 60 years. The International Federation of Gynecology and Obstetrics (FIGO) stage distribution of the cervix cancer patients was as follows: stage I - 34 cases, stage IIA or IIB - 134, stage III - 118 patients, and stage IVA - 31 cases.

All patients received primary radiotherapy and no previous treatment. For each of the 314 patients, insertions were placed correctly, and the brachytherapy course was completed uneventfully. During brachytherapy, the sleeve dislocated in two patients due to suture failure, and it was reinserted with ultrasonography guidance. In $48(15.1 \%)$ patients, intra-operative ultrasonography was used to guide sleeve insertion.

Of the 317 patients, only one patient $(0.3 \%)$ had uterine perforation. In that case, the sleeve was inserted into the uterine cavity without ultrasonography guidance. Uterine perforation through the posterior wall was diagnosed on planning CT scan. The applicator was removed, and the patient was treated in the hospital with a broad spectrum of antibiotics. The patient was 67 years old with stage IIIB that involved all upper $1 / 3$ of the vagina; thus, the ectocervix was fully distorted. The second insertion attempt was not applied. The patient was given extra external RT. Except for one patient, the sleeve was removed uneventfully after the third session of brachytherapy. Although hypothetically, the sleeve could be a conduit for infection, there was no complications associated with infection.

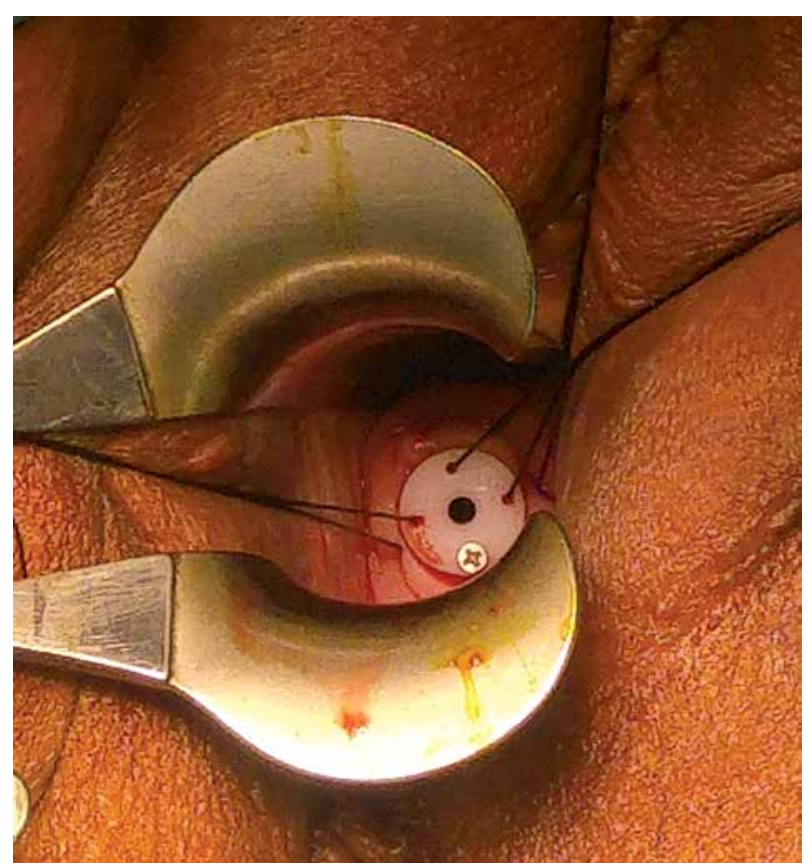

Fig. 1. The Smit sleeve is inserted into the cervical os and sutured in place prior to the first brachytherapy

\section{Discussion}

Intracavitary brachytherapy is the sine qua non of the treatment of local advanced cervical cancer with primer chemoradiation therapy. Brachytherapy requires accurate placement of an applicator into the uterine cavity through the cervical canal. However, reaching the uterine cavity through a cervical canal that was distorted by cancer involves some risk of complications. This procedure has often been performed by relying only on the experience of operating physician. Intracavitary brachytherapy requires commonly three treatment cycle, and the placement of applicator is traditionally performed by a radiation oncologist. As the treatment schedule consists of three separate insertions, every placement of the applicator has the potential for additional risk of uterine perforation. Uterine perforation has many potential harmful effects on the treatment; therefore, strategies are needed to reduce inaccurate positioning of the intracavitary applicator. The use of Smit sleeve before brachytherapy is a technique, which facilitates subsequent placement of tandem. The accurate placement of Smit sleeve eliminates the risk of malposition of tandem in the subsequent insertions [11].

In a study that evaluated 428 applications of tandem in 219 patients, uterine perforation was detected in $13(3.0 \%)$ applications in $10(4.6 \%)$ patients. In that study, all applications were performed by a radiation oncologist alone, except in 3 cases, where the procedure was conducted in cooperation with a gynecologic oncologist. Although the author of that study indicated that their incidence of perforations as one of the lowest reported in the literature, multiple applications increased the risk of perforation per patient [12]. Therefore, using the Smit sleeve technique facilitates subsequent insertions and decrease the risk of perforation in total. In our study, the Smit sleeve provid- 
ed safe and effective guidance for subsequent insertions resulting in decreased rates of perforations and misplacement of applicators.

Barnes et al. prospectively compared radiation oncologists' clinical impressions of satisfactory tandem placement with actual tandem placement as determined by pelvic CT. The incidence of CT-detected uterine perforation was $13.7 \%(17 / 124)$. Furthermore, CT-detected perforation in $8.2 \%(8 / 98)$ of insertions was shown, where the radiation oncologist was clinically confident of correct tandem placement [6]. In another study that obtained data from 200 patients treated with CT-based brachytherapy, $8.5 \%$ of patients had uterine perforation [13]. Post-procedure CT scans are routinely performed at our center, and we detected only one uterine perforation. The incidence of uterine perforations at our institute was observed as the lowest reported in the literature. However, several factors played a role in this result.

Firstly, all insertions have been applied by gynecologic oncologists in our center. Uterine perforation is a complication that is well recognized by all gynecologists. Each gynecologist has experienced this complication at least once in their professional practice. Numerous reports have suggested that trainees are more likely to perforate the uterus compared with experienced surgeons [14]. Passing through a cervical canal distorted by cancer and fibrosis from radiotherapy is a challenge. Therefore, physicians performing tandem placement must adequately trained. The reported studies in the literature typically did not describe how many insertions were performed by either gynecologic oncologists or radiation oncologist. Although the positioning of tandem is commonly performed by a radiation oncologist, differences in insertion techniques could also influence physician's experience [15].

Secondly, we routinely assess uterine position with ultrasonography before application at the outpatient clinic, and generally, guidance of ultrasonography is applied when detecting any resistance during the application procedure. In our clinic, the decision whether or not to guide with ultrasonography is individualized and depends on the hysterometry resistance during cervical dilatation. Although traditionally, the tandem applicator is placed through the cervical os without ultrasonography guidance. A meta-analysis by Sapienza et al. reported that the rate of uterine perforation per insertion in patients who received ultrasonography-guided brachytherapy insertion was $90 \%$ lower than with unguided insertion, and the pooled perforation rate per patient without guidance was $16.67 \%$ (95\% CI: $10.01-26.45 \%)$ vs. $2.54 \%$ (95\% CI: $1.21-5.24 \%$ ) with image guidance [15]. Ultrasonography guidance during brachytherapy is associated with a higher rate of correct insertion of applicator and a lower rate of uterine perforation [16]. In the current study, the only perforation occurred when applicator was placed without ultrasonography guidance in the patient with distorted cervix by stage IVA cervical cancer. Therefore, brachytherapy was abandoned, and external radiotherapy was delivered instead for this patient. The cervical sleeve was inserted accurately to all other patients who applied to our institute for brachytherapy. The procedure did not fail, except for the only case with perforation.
Accurate cervical sleeve placement facilitates correct tandem placement. A previous study reported that optimal tandem placement improves survival and pelvic control among patients with cervical cancer [17].

One concern may emerge about the impact of Smit sleeve usage on dosimetric planning. Mehta et al. reported that the Smit sleeve does have a dosimetric impact, which should be considered when a tandem and ovoids system is used. However, the use of a Smit sleeve with a tandem and ring applicators system does not have the same consequence on the applicators' geometry [18].

The current study was limited by its retrospective nature and the inherent biases of retrospective studies. Another potential limitation might be underreporting of some outcomes. Despite these limitations, a notable strength of our study was that it resulted in the largest series of complications to report due to applicator insertion. Another strength of our study was that after insertion, all applicators were reviewed with CT-scans to confirm their correct positioning.

\section{Conclusions}

Uterine perforation during the placement of tandem can have potentially catastrophic consequences for cervical cancer patients. Inaccurate applicator placement can be associated with worse survival rates. The best outcomes are achieved when each patient is evaluated in a multidisciplinary setting and the team of clinicians, including radiation oncologists, surgeons, anesthesiologists, and other specialists. Experienced hands, the use of a cervical sleeve, and ultrasonography guidance can all help to minimize the risk of perforation, providing the accurate placement of applicators. This technique has the potential to improve local control and prevent dissemination in cervical cancer patients.

\section{Disclosure}

The authors report no conflict of interest.

\section{References}

1. Viswanathan AN, Moughan J, Small W et al. The quality of cervical cancer brachytherapy implantation and the impact on local recurrence and disease-free survival in radiation therapy oncology group prospective trials 0116 and 0128 . Int J Gynecol Cancer 2012; 22: 123-131.

2. Rose PG, Ali S, Watkins E et al. Long-term follow-up of a randomised trial comparing concurrent single agent cisplatin, cisplatin-based combination chemotherapy, or hydroxyurea during pelvic irradiation for locally advanced cervical cancer: a Gynecologic Oncology Group Study. J Clin Oncol 2007; 25: 2804-2810.

3. Han K, Milosevic M, Fyles A et al. Trends in the utilisation of brachytherapy in cervical cancer in the United States. Int J Radiat Oncol Biol Phys 2013; 87: 111-119.

4. Liu R, Wang X, Tian J et al. High dose rate versus low dose rate intracavity brachytherapy for locally advanced uterine cervix cancer. Cochrane Database Syst Rev 2014; 10: CD007563.

5. Schaner PE, Caudell JJ, De Los Santos JF et al. Intraoperative ultrasound guidance during intracavitary brachytherapy applicator placement in cervical cancer: The University of Ala- 
bama at Birmingham experience. Int J Gynecol Cancer 2013; 23: 559-566.

6. Barnes EA, Thomas G, Ackerman I et al. Prospective comparison of clinical and computed tomography assessment in detecting uterine perforation with intracavitary brachytherapy for carcinoma of the cervix. Int J Gynecol Cancer 2013; 17: 821-826.

7. Bahadur YA, Eltaher MM, Hassouna AH et al. Uterine perforation and its dosimetric implications in cervical cancer high-dose-rate brachytherapy. J Contemp Brachytherapy 2015; 7: 41-47.

8. Heinemann K, Reed S, Moehner S et al. Risk of uterine perforation with levonorgestrel-releasing and copper intrauterine devices in the European Active Surveillance Study on Intrauterine Devices. Contraception 2015; 91: 274-279.

9. Smit BJ, van Wijk AL. An improved, disposable indwelling intrauterine tube ("smit sleeve") not requiring retaining stitches for brachy-radiotherapy for carcinoma of the cervix. Eur J Gynaecol Oncol 2013; 34: 289-290.

10. Smit BJ. Design features of the indwelling intrauterine tube for high dose rate intracavitary therapy for carcinoma of the cervix and some hints on its optimal use. Br J Radiol 1993; 66: 1042-1043.

11. Tyrie LK, Hoskin PJ. Intrauterine high dose rate afterloading brachytherapy: experience of fractionated therapy using a cervical sleeve technique. Clin Oncol 1996; 8: 376-379.

12. Segedin B, Gugic J, Petric P. Uterine perforation-5-year experience in 3-D image guided gynaecological brachytherapy at Institute of Oncology Ljubljana. Radiol Oncol 2013; 47: 154-160.

13. Onal C, Guler OC, Dolek Y et al. Uterine perforation during 3-dimensional image-guided brachytherapy in patients with cervical cancer: Baskent University experience. Int J Gynecol Cancer 2014; 24: 346-351.

14. Shakir F, Diab Y. The perforated uterus. Obstet Gynaecol 2013; 15: 256-261.

15. Sapienza LG, Jhingran A, Kollmeier MA et al. Decrease in uterine perforations with ultrasound image-guided applicator insertion in intracavitary brachytherapy for cervical cancer: A systematic review and meta-analysis. Gynecol Oncol 2018; 151: 573-578.

16. Akbas T, Ugurluer G, Acil M et al. Intraoperative sonographic guidance for intracavitary brachytherapy of cervical cancer. J Clin Ultrasound 2018; 46: 8-13.

17. Corn BW, Hanlon AL, Pajak et al. Technically accurate intracavitary insertions improve pelvic control and survival among patients with locally advanced carcinoma of the uterine cervix. Gynecol Oncol 1994; 53: 294-300.

18. Mehta S, Farnia B, De La Zerda et al. Dosimetric effects of the Smit sleeve on high-dose-rate brachytherapy tandem and ovoids plans for patients with locally advanced cervical cancer. J Contemp Brachytherapy 2019; 11: 584-588. 